\title{
Internationale Marktforschung
}

\author{
Stephan Broda
}

\section{Zusammenfassung}

Unternehmen, die international agieren bzw. agieren möchten, müssen die Funktionsweisen der fremden Märkte kennen und ihre Geschäftspraktiken entsprechend einstellen. Internationale Marketingstrategien zeichnen sich durch hohe Komplexität aus. Der Beitrag gibt einen Überblick über die Bedeutung und wesentlichen Elemente der internationalen Marktforschung sowie über deren Zukunftstrends.

\section{Abstract}

Companies already acting internationally or going international need to understand how international markets function and how the need to adjust their business practices. Yet international marketing strategies are characterised by great complexity. The article gives an overview of the meaning of international market research, its core elements as well as trends for the future.

\section{Warum internationale Marktforschung?}

Die richtige Einschätzung des internationalen Marktgeschehens ist für jedes Unternehmen eine große Herausforderung, da die Implementierung globaler Marketingstrategien durch hohe Komplexität, zeitliche Unsicherheiten sowie beträchtliche Kosten gekennzeichnet ist. Im Rahmen der Risikominimierung setzt hier die internationale Marktforschung an, die eine entscheidende Rolle bei der Vorbereitung von Marketingentscheidungen spielt. Dabei erfüllt die internationale Marktforschung folgende Hauptaufgaben (Homburg/ Krohmer 2003, S. 920; Broda 2005, S. 101 ff.; Knapp/ Wachter 1998, S. 26):

- Sie liefert die empirische Datenbasis, um die Expansion in renditeträchtige Auslandsmärkte zu beschleunigen oder geeignete Übernahmekandidaten (Produkte, Firmen) zu identifizieren.

- Die internationale Marktforschung ermöglicht die Früherkennung von Marktveränderungen (TrendScout-Funktion), welche später auch für das eigene Unternehmen oder den nationalen Markt von Bedeutung sein können.

- Die Beobachtung internationaler Produktmärkte führt zu Lerneffekten, da ausländische Konkurrenzaktivitäten analysiert (Best Practice, Benchmarking) und für das eigene Unternehmen nutzbar gemacht werden.

- Internationale ökonomische sowie politische Veränderungen haben Auswirkungen auf den nationalen Markt (z. B. Asienkrise oder 11.09.2001). Hier gilt es adäquat zu reagieren.

\section{Anforderungen an die internationale Marktforschung}

Im internationalen Bereich stellt sich insbesondere die Frage, inwieweit Marktforschungsmethoden über verschiedene Kulturkreise hinweg gültig sind. So treffen beispielsweise in westlichen Kulturkreisen die Konsumenten individuelle Kaufentscheidungen, hingegen spielen in östlichen Kulturen Gruppenentscheidungen eine wichtigere Rolle (Homburg/Krohmer 2003, S. 920). In der Praxis lässt sich jedoch feststellen, dass zwei Drittel aller internationalen Marktforschungsstudien in den in Abbildung 1 dargestellten Ländern durchgeführt werden, so dass die Problematik internationaler Gültigkeit der Forschungsergebnisse nicht überbewertet werden sollte.

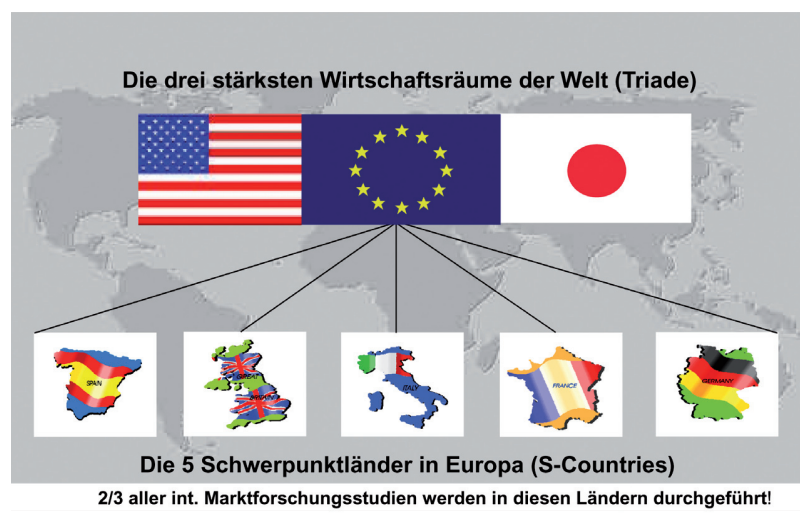

Abb. 1: Geografische Schwerpunkte internationaler Marktforschung

Um auch im internationalen Bereich die üblichen Gütekriterien wie Reliabilität und Validität zu erfüllen und darüber hinaus kulturübergreifend vergleichbare Daten zu erheben, ist es zwingend erforderlich, ein einheitliches Studiendesign (Untersuchungsinhalt, Zielgruppen, 
Methodik, Fragebogen, Vercodung, Auswertung und Interpretation) zu gewährleisten (Knapp/Wachter 1998, S. 27).

Dabei ist es besonders wichtig, dass die Befragten in den verschiedenen Ländern die Bedeutung von Fragen und Ratingskalen identisch interpretieren (Verständnisäquivalenz). Des weiteren ist sicherzustellen, dass die Respondents in den verschiedenen Ländern bei Gleichheit der relevanten Bedingungen auch gleich antworten (Beantwortungsäquivalenz). Hier entstehen vor allem folgende Probleme (Knapp/Wachter 1998, S. 29 f.; Homburg/Krohmer 2003, S. 921 f.):

- Soziale Wünschbarkeit: Bezüglich der sozialen Erwünschtheit von Antworten ist es beispielsweise in den USA durchaus üblich, sich sehr positiv über den persönlichen Erfolg zu äußern, wohingegen dies in Frankreich ein eher weniger akzeptiertes soziales Verhalten darstellt.

- Höflichkeitsbias: Im Kommunikationsverhalten ist zu beachten, dass die Bereitschaft zur deutlichen Kritikäußerung je nach Land unterschiedlich hoch ausgeprägt ist. Insbesondere in Ostasien besteht die Neigung, den Interviewer nicht zu verärgern und daher stets zustimmende Antworten zu geben.

- Extremer Antwortstil: Bei Skalenabfragen können verstärkt die Extrempunkte der Skala oder nur die Mitte besetzt sein. Ebenfalls ist häufig eine Ja/NeinSage-Tendenz zu beobachten.

- Allgemeiner Sprachgebrauch: Bei der Gestaltung von Fragen ist ein nach Ländern unterschiedlicher Sprachgebrauch zu berücksichtigen. So bietet die deutsche Sprache im allgemeinen die Möglichkeit zu präziseren Formulierungen, als beispielsweise die US-amerikanische.

\section{Organisation der internationalen Marktforschung}

In den meisten Fällen wird die internationale Marktforschung in einem Unternehmen zentral gesteuert, d. h. benötigte Daten der Sekundärmarktforschung und Wettbewerbsanalyse werden an einer Stelle zusammengetragen und Primärstudien von der Unternehmenszentrale aus koordiniert. Dies hat vor allem die folgenden Vorteile (Doole/Lowe 2001, S. 23 ff.):

- Die zentrale Durchführung von Marktforschungsstudien sichert die Vergleichbarkeit der Länderergebnisse durch ein identisches Studiendesign.

- Es kommen nur die vorab festgelegten, einheitlichen Markt-, Produkt- und Zielgruppendefinitionen zur Anwendung.

- Kommunikations- und Informationsprozesse werden durch eine zentrale Anlaufstelle wesentlich vereinfacht. Dies führt zu erhöhter Transparenz des gesamten Studienablaufs.

- Der Informations- und Know-how-Transfer zu den Tochtergesellschaften wird erhöht und verbessert somit die Akzeptanz des gesamten Projektes. Das »Not Invented Here« (NIH)-Syndrom wird vermieden.
- Nicht zuletzt führt die zentrale Vorgehensweise zu signifikanten Zeitgewinnen sowie zu Budgetersparnissen für die Tochtergesellschaften, da zentral in Auftrag gegebene Studien i. d. R. auch zentral bezahlt werden.

Ein Unternehmen nimmt i. d. R. bei der Durchführung von internationalen Marktforschungsstudien die Dienste von internationalen Marktforschungsagenturen in Anspruch. Größere Agenturen, von denen drei in Abbildung 2 beispielhaft dargestellt sind, verfügen meist über eigene Niederlassungen in den meisten Ländern der Erde.
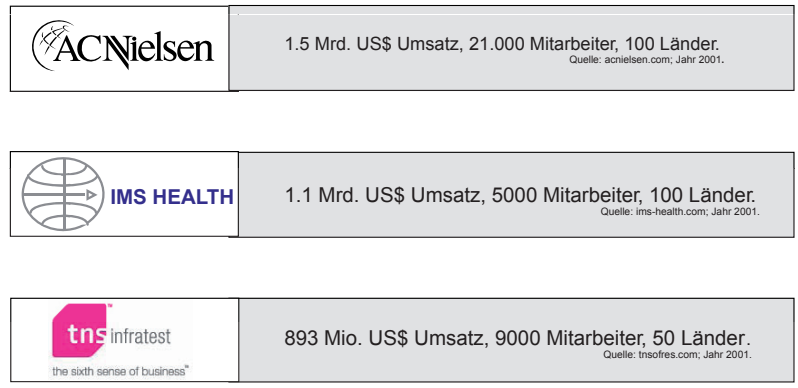

Abb. 2: Internationale Marktforschungsagenturen

\section{Hauptbereiche internationaler Marktforschung}

Wie auch im nationalen Bereich stellen Sekundär- und Primärmarktforschung sowie die Wettbewerbsanalyse das Fundament jeder Marktforschungsabteilung dar. Allerdings gibt es in den Aufgaben der einzelnen Bereiche einige Unterschiede, die nachfolgend beschreiben werden (Broda 2005, S. 101 ff.).

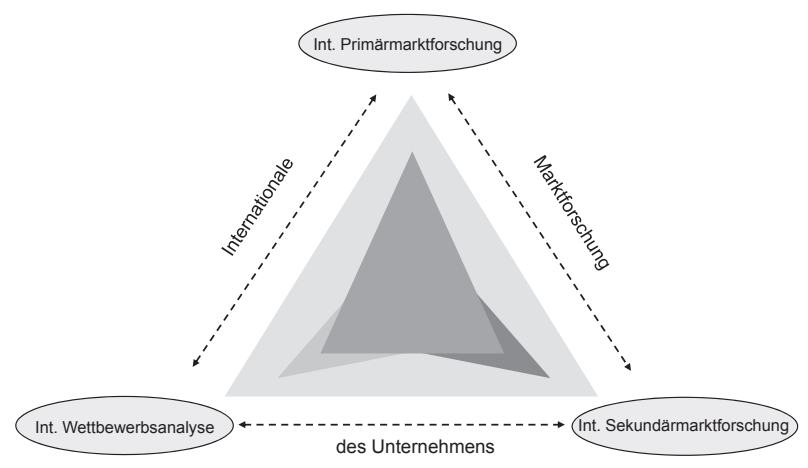

Abb. 3: Die drei Hauptbereiche internationaler Marktforschung

\subsection{Sekundärmarktforschung}

Internationale Sekundärforschung interpretiert bereits vorhandene Daten und stellt deren internationale Vergleichbarkeit her. Im Hinblick auf Quellen für Sekundärdaten sind im internationalen Marketing zwei Kategorien zu unterscheiden (Kotler/Bliemel 2001, S. $381 \mathrm{ff}$. Rogge 1999, S. 79 ff.):

- Nationale Quellen: Statistische Ämter des Landes, Verbände, wissenschaftliche Institutionen, Nationale Verzeichnisse (z. B. »Rote Listen«). 
- Internationale Quellen: Amtliche Statistiken der Worldbank, United Nations Population Fund, International Monetary Fund (IWF).

Eine weitere zentrale Aufgabe internationaler Sekundärmarktforschung ist die konzernweite Harmonisierung und Durchsetzung von Datenstandards und Marktdefinitionen. Bei der Verwendung von Sekundärdaten im internationalen Bereich können spezifische Probleme auftreten. Dabei sind insbesondere zu erwähnen (Homburg/Krohmer 2003, S. 923; Peter/Donnelly Jr. 2002, S. $47 \mathrm{f}$.$) :$

- Genauigkeit der Daten: Bezüglich der Qualität von vorhandenem sekundärstatistischen Material gibt es zwischen den einzelnen Ländern große Unterschiede.

- Länderübergreifende Vergleichbarkeit der Daten: Oftmals lassen sich Sekundärdaten aus verschiedenen Ländern schwer miteinander vergleichen, da sie unterschiedlich gegliedert sind und auf verschiedenen Erhebungsinstrumenten basieren.

\subsection{Primärmarktforschung}

Einen Überblick über die Vielzahl möglicher internationaler Studien liefert Abbildung 4. Im internationalen Bereich wird, allein schon aus praktischen Gründen, am häufigsten die Befragung als »klassische Erhebungsmethode « eingesetzt. Auch hier sind identische Erhebungsmethoden (z. B. Face-to-Face), über alle an der Studie beteiligten Länder das wichtigste Kriterium (Doole/Lowe 2001, S. 278 f.).

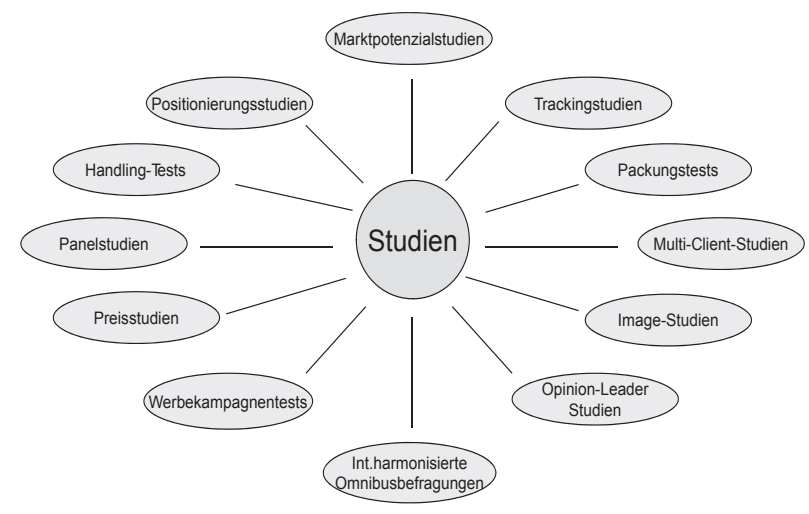

Abb. 4: Internationale Primärmarktforschungsstudien

Analysiert man hier die schriftliche, telefonische und die Face-to-Face Befragung, so ergeben sich für den internationalen Einsatz folgende Vor- und Nachteile (Berekoven et al. 2004, S. 94 ff.; Lüttgens 1998, S. 59 f.; Böhler 2004, S. 64 f.):

\subsubsection{Schriftliche Befragung}

Die niedrigen Durchführungskosten sind gerade im internationalen Bereich von Vorteil, da hier die Möglichkeit zur Einbeziehung auch geographisch weit gestreuter Befragter besteht. Auch wirkt sich die Vermeidung von länderspezifischen Interviewereinflüssen positiv aus. Als echter Nachteil hingegen muss die unterschiedlich hohe Akzeptanz von schriftlichen Befragungen in den einzelnen Ländern gesehen werden, die zu sehr unter- schiedlichen Rücklaufquoten führen kann. Auch sind Verzögerungen im internationalen Postversand entsprechend einzukalkulieren.

\subsubsection{Telefonbefragung}

Internationale Telefonbefragungen lassen sich zentral, d. h. aus dem Heimatland, schnell und sicher durchführen. Man ist zeitlich sehr flexibel und kann mit sehr geringen Abbrecherquoten rechnen. Leider steht dem ein relativ hoher Koordinationsaufwand gegenüber, insbesondere wenn eine »kalte « Anwahl nicht möglich ist, also vorab Terminvereinbarungen mit dem Respondent getroffen werden müssen. Die Problematik unterschiedlicher Zeitzonen, teilweise unzureichender Telefondichte und oftmals schlechter Netzqualität sollte nicht unterschätzt werden.

\subsubsection{Face-to-Face-Interview}

Da bei Face-to-Face-Interviews i. d. R. lokale Interviewer eingesetzt werden, ist die Wahrscheinlichkeit kulturbedingter Missverständnisse eher gering. Leider mangelt es oft an geschulten Interviewern vor Ort und die ohnehin hohen Kosten schlagen im internationalen Bereich besonders zu Buche (Training der Interviewer, Reisekosten, Koordination).

\subsection{Wettbewerbsanalyse (Business Intelligence)}

Unter internationaler Wettbewerbsanalyse versteht man die strukturierte, systematische und dauerhafte Beobachtung von Konkurrenzunternehmen zur Unterstützung der Entscheidungsfindung in der Marketingplanung. Dabei stellt das Auffinden potenzieller Übernahme-, Lizenz- oder Kooperationskandidaten einen wesentlichen Beitrag dar. Es werden Firmenprofile (SWOT-Analysen, Patentsituation, Produktportfolio, Pipeline-Situation) erstellt und im Sinne der nachhaltigen Sicherung von Erfolgspotenzialen ein »Competitive Monitoring « aufgebaut (Keegan/Green 2000, S. 124 ff.).

\section{Durchführung einer internationalen Marktforschungsstudie}

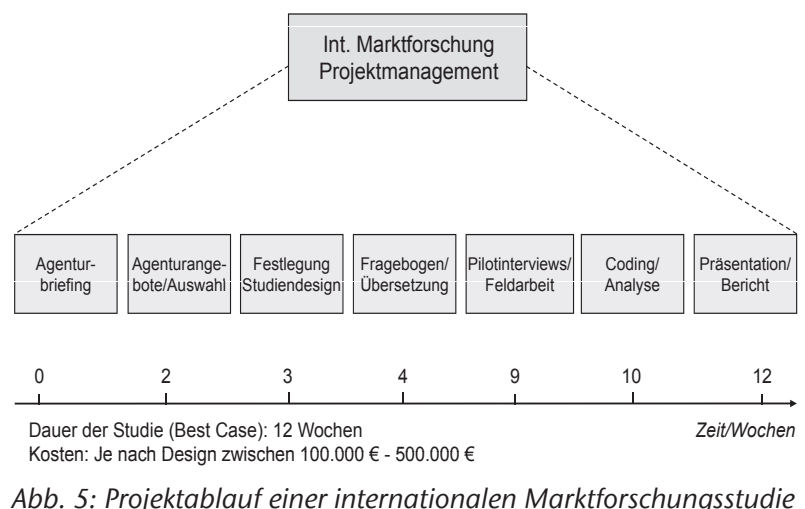

Im Hinblick auf die Sicherstellung einer hohen Qualität internationaler Marktforschungsstudien sind bei deren Durchführung die nachfolgenden Punkte unbedingt zu 
beachten (Homburg/Krohmer 2003 S. 922 f.; Backhaus et al. 1998, S. 114 ff.; Cateora/Graham 2001, S. 82 ff.):

- In die Konzeption eines internationalen Marktforschungsprojektes sollten von vornherein Personen mit Verständnis für die verschiedenen Landeskulturen mit einbezogen werden.

- Bei der Verwendung standardisierter Fragebögen in mehreren Ländern empfiehlt es sich, den Fragebogen von einer zweisprachigen Person kontrollieren zu lassen und mit der englischen Masterversion abzugleichen.

- Sorgfältige Pretests, möglichst in mehreren der beteiligten Länder, sind im internationalen Marketing noch wichtiger als bei rein nationaler Marktforschung.

- Es ist zu berücksichtigen, dass die verschiedenen Datenerhebungsmethoden (z. B. Face-to-Face-Befragung vs. Telefoninterview) nicht in allen Ländern die gleiche Akzeptanz bei den Befragten aufweisen.

- Es ist bei der Planung einer internationalen Mehrländerstudie immer ausreichend Zeit und Budget bereitzustellen. Als »rule of thumb « sollten ein Zeitrahmen von zwölf Wochen und Kosten ab 100.000 Euro eingeplant werden.

\section{Zukunftstrends in der internationalen Marktforschung}

Vor allem aus Kostengründen zeichnen sich drei wesentliche Trends ab. Zum ersten wird die Online-Marktforschung (Internetmarktforschung) sehr starke Zuwachsraten verzeichnen, da sie die Gestaltungsmöglichkeiten des Face-to-Face-Interviews mit den Kostenvorteilen der Telefonumfrage kombiniert. Zweitens wird es eine Bewegung weg von der reinen Institutsmarktforschung hin zur »Universitätsmarktforschung « über Partnerhochschulen im internationalen Umfeld geben und drittens wird sich die Gefahr des kompletten Outsourcings der internationalen Marktforschung an Agenturen erhöhen. Lediglich die Koordination wird in der Marketingabteilung der jeweiligen Firmen verbleiben. Dennoch wird auch in Zeiten enger Budgets die »klassische « internationale Marktforschung eine Zukunft haben.

\section{Literatur}

Backhaus, K./Büschen, J./Voeth, M., Internationales Marketing, 2. Aufl., Stuttgart 1988

Berekoven, L./Eckert, W./Ellenrieder, P., Marktforschung, 10. Aufl., Wiesbaden 2004

Böhler, H., Marktforschung, 3. Aufl., Stuttgart, 2004

Broda, S., Marketing-Praxis, 2. Aufl., Wiesbaden 2005

Cateora, P. R./Graham, J. L., International Marketing, 11th Edition, New York 2001

Doole, I./Lowe, R., International Marketing Strategy, 3rd Edition, 2001, Massachusetts 2001

Homburg, C./Krohmer, H., Marketingmanagement, 1. Aufl., Wiesbaden 2003

Keegan, W. J./Green, M. C., Global Marketing, 2nd Edition, New York 2000

Knapp, F. D./Wachter, B., Internationale Marktforschung Lediglich eine Frage der Segmentierung?, in: Planung \& Analyse, 6/1998

Kotler, P./Bliemel, F., Marketing Management, 10. Aufl., Stuttgart 2001

Lüttgens, M. R., Marketing Forschung, 1. Aufl., Bern 1998

Peter, J. P./Donnelly Jr., J. H., A Preface to Marketing Management, 9th Edition, Illinois 2002

Rogge, H.-J., Sekundärerhebung (Informationsquellen), in: Pepels, W., Moderne Marktforschung, Neuwied 1999

\section{Autor}

Prof. Dr. Stephan Broda

Technische Fachhochschule Wildau

Tel. +493375 508-869

stephan.broda@tfh-wildau.de 